\title{
Delithiated states of layered cathode materials: doping and dispersion interaction effects on the structure
}

\author{
Roman Eremin ${ }^{1, *}$, Pavel Zolotarev ${ }^{1}$, and Ivan Bobrikov ${ }^{2,3}$ \\ ${ }^{1}$ Samara Center for Theoretical Materials Science, Samara University, Samara, Russia \\ ${ }^{2}$ Frank Laboratory of Neutron Physics, Joint Institute for Nuclear Research, Dubna, Russia \\ ${ }^{3}$ Saratov State University, Saratov, Russia
}

\begin{abstract}
Here we present results of density functional theory (DFT) study of delithiated structures of layered $\mathrm{LiNiO}_{2}\left(\mathrm{LNO}, \mathrm{Li}_{12} \mathrm{Ni}_{12} \mathrm{O}_{24}\right.$ model) cathode material and its doped analogue $\mathrm{LiNi}_{0.833} \mathrm{Co}_{0.083} \mathrm{Al}_{0.083} \mathrm{O}_{2}\left(\mathrm{~N}_{10} \mathrm{C}_{1} \mathrm{~A}_{1}\right.$, $\mathrm{Li}_{12} \mathrm{Ni}_{10} \mathrm{CoAlO}{ }_{24}$ model). The paper is aimed at independent elucidation of doping and dispersion interaction effects on the structural stability of cathode materials studied. For this purpose, the LNO and $\mathrm{N}_{10} \mathrm{C}_{1} \mathrm{~A}_{1}$ configurational spaces consisting of 87 and 4512 crystallographically independent configurations (obtained starting from $2 \times 2 \times 1$ supercell of R$3 \mathrm{~m}$ structure of LNO) are optimized within a number of DFT models. Based on a comparison of the calculated dependencies for the lattice parameters with the results of in situ neutron diffraction experiments, the most pronounced effect of cathode material stabilization is due to the dispersion interaction. In turn, the doping effect is found to affect cathode structure behavior at the latest stages of delithiation only.
\end{abstract}

\section{Introduction}

At present, rechargeable Li-ion batteries have become an important component of modern electronic devices (portable electronics, variety of vehicle, medical equipment, etc.). In an effort to improve their performance both theoreticians and experimentalists investigate structure and properties of electrode materials as well as the ways to modify/improve the working characteristics [1-4]. Among the theoretical approaches, a number of computer simulation techniques, such as density functional theory (DFT) calculations, are applied for predicting properties of the electrode materials [5].

In order to perform a comprehensive modeling, it is necessary to take into account disordering of the electrode structure. Moreover, different types of corrections are needed to improve descriptive power of a DFT model, e.g. the dispersion interactions for layered cathode materials [6]. There are two types of approaches to handle the disordering: modeling of a limited number of random configurations [7] or employing fast screening

*Corresponding author: eremin_roman@inbox.ru 
methods for all possible structure realization [8]. The issues of structural stability, ion migration hindrance and voltage dependence versus deintercalation of the mobile ions can be efficiently simulated as well as cation/anion doping effect on the characteristics [8, 9]. Modern experimental techniques allow tracking structural changes of the electrodes during charge/discharge processes. For the commercial $\mathrm{LiNi}_{0.8} \mathrm{Co}_{0.15} \mathrm{Al}_{0.05} \mathrm{O}_{2}$ (NCA) cathode studies were carried out by means of in situ x-ray [10] and neutron [11,12] diffraction.

Previously, the full $\mathrm{LiNiO}_{2}$ (LNO) and NCA configurational spaces were set using topological approach and studied by means of the DFT calculations and machine learning algorithms [13]. In the scope of the current research, the topological approach is applied for sampling of the configurational space of the $\mathrm{LiNi}_{0.833} \mathrm{Co}_{0.083} \mathrm{Al}_{0.083} \mathrm{O}_{2}\left(\mathrm{~N}_{10} \mathrm{C}_{1} \mathrm{~A}_{1}\right)$ cathode. The work is aimed at the computational study of the effect of structural stabilization of the LNO-based cathodes doping and dispersion interactions. The approach is based on the comparison of results of computer modeling and in situ neutron diffraction data [12].

\section{Methods}

The step-by-step methodology of sampling of the configurational spaces for $\mathrm{N}_{10} \mathrm{C}_{1} \mathrm{~A}_{1}$ was used as described in [13] for $\mathrm{N}_{9} \mathrm{C}_{2} \mathrm{~A}_{1}$ using the ToposPro package [14]. For the current study, change of the composition (from $\mathrm{N}_{9} \mathrm{C}_{2} \mathrm{~A}_{1}$ to $\mathrm{N}_{10} \mathrm{C}_{1} \mathrm{~A}_{1}$ ) is reasonable from the computational complexity point of view. In situ neutron diffraction experiments were carried out during a charge-discharge process using the Real Time Diffractometer and the High Resolution Fourier Diffractometer (IBR-2, FLNP, JINR). The procedure of collection of neutron diffraction patterns and structure refinement was described [12] in details.

The obtained configurational spaces were optimized within the DFT relaxation using the projector-augmented wave method and Perdew-Burke-Ernzerhof (PBE) exchangecorrection functional within the generalized gradient approach (GGA) as implemented in the Vienna Ab Initio Simulation Package (VASP) [15]. At the next stage of analysis, the DFT-D3 method [16] was applied as implemented in VASP (noticed hereafter by abbreviation ' $v d W$ ' stands for 'van der Waals' forces). The further model refinement was carried out in the spin-restricted mode of DFT. The spin-polarized (SP) models were considered with non-magnetic (NM), ferromagnetic (FM), and two antiferromagnetic (AFM) initial guesses (magnetic moment of $2 \mu_{B}$ was associated with each Ni atom). Final improvement of the SP models was the Hubbard approach for descriptions of the localized electron states ( $d$ orbitals) of transition metal (Ni), $U=6.0 \mathrm{eV}$ [17].

The DFT modeling allowed us to perform averaging of the mentioned structural parameters of the cathode material over the configurational space [18]:

$$
y(x)=\Sigma_{x} y_{i} \omega_{i} \exp \left(-E_{i} / k T\right) / \Sigma_{x} \omega_{i} \exp \left(-E_{i} / k T\right),
$$

where $y(x)$ is the mean value of a lattice parameter at a certain delithiation level (in $\AA$ ), $x$; $\omega_{i}, y_{i}$ (in $\AA$ ), $E_{i}$ (in eV/atom) have meanings of the statistical weight of a certain configuration, the current parameter value and the normalized free energy value of $i^{\text {th }}$ configuration, respectively; the Boltzmann constant $k=8.617 \cdot 10^{-5} \mathrm{eV} / \mathrm{K}$ and $T$ is the temperature (in K). The summation in formula (1) is performed over the whole set of studied configurations corresponding to a certain delithiation level, $x\left(\mathrm{Li}_{1-x} \mathrm{MeO}_{2}, x=0 \div 1\right)$. 


\section{Results}

\subsection{Configuration space sampling}

The LNO configurational space consisting of 87 entries with respect to Li content and its distribution in the model cell was obtained during full delithiation [13]. For each independent $\mathrm{Li}$ disposition the substitution of two $\mathrm{Ni}$ atoms (over twelve possible atomic positions in the sublattice) by dopants (one $\mathrm{Al}$ and one Co atoms) leads to totally 132 possible configurations. Thus, 11484 configurations were obtained as the reducible configurational space of $\mathrm{N}_{10} \mathrm{C}_{1} \mathrm{~A}_{1}$. After that, 4512 configurations (instead of 20760 ones in case of $\mathrm{N}_{9} \mathrm{C}_{2} \mathrm{~A}_{1}$ model of NCA [13]) were found to be structurally independent at the topological selection stage. The counts of the obtained configurations of $\mathrm{LNO}$ and $\mathrm{N}_{10} \mathrm{C}_{1} \mathrm{~A}_{1}$ are listed in Table 1 for each delithiation level, $x$. The statistical weights of all independent configurations were collected for the subsequent averaging procedure.

Table 1. Statistics of the independent configurations versus delithiation, $x$, for $\mathrm{LNO}$ and $\mathrm{N}_{10} \mathrm{C}_{1} \mathrm{~A}_{1}$.

\begin{tabular}{|c|c|c|c|c|c|c|c|c|c|c|c|c|c|}
\hline $\boldsymbol{x}$ & $\mathbf{0}$ & $\mathbf{1 / 1 2}$ & $\mathbf{1 / 6}$ & $\mathbf{1 / 4}$ & $\mathbf{1 / 3}$ & $\mathbf{5 / 1 2}$ & $\mathbf{1 / 2}$ & $\mathbf{7 / 1 2}$ & $\mathbf{2 / 3}$ & $\mathbf{3 / 4}$ & $\mathbf{5 / 6}$ & $\mathbf{1 1 / 1 2}$ & $\mathbf{1}$ \\
\hline $\mathrm{LNO}$ & 1 & 1 & 3 & 6 & 11 & 13 & 17 & 13 & 11 & 6 & 3 & 1 & 1 \\
\hline $\mathrm{N}_{10} \mathrm{C}_{1} \mathrm{~A}_{1}$ & 3 & 21 & 88 & 258 & 547 & 849 & 980 & 849 & 547 & 258 & 88 & 21 & 3 \\
\hline
\end{tabular}

\subsection{DFT-based geometry optimization}

The statistically averaged dependencies $(T \rightarrow \infty)$ of $a$ and $c$ lattice parameters of the LNO crystal structure are shown in Fig. 1. Obviously, prediction of the GGA-PBE model on the $c$ parameter strongly deviates in comparison to the other DFT models that take into account dispersion interactions and results in structural stabilization (non-monotonic behavior of $c$ parameter). Thus the obtained results are in a good agreement with the fact that dispersion correction becomes important at high deintercalation levels [6]. The most important observation at the current stage of analysis is the fact that relative variances of the $a$ and $c$ lattice parameter values that were calculated using the dispersion corrected models $(+\mathrm{vdW},+\mathrm{vdW}+\mathrm{SP}$ and $+\mathrm{vdW}+\mathrm{SP}+\mathrm{U})$ do not exceed $1.8 \%$ and $1.4 \%$, respectively.

(a)

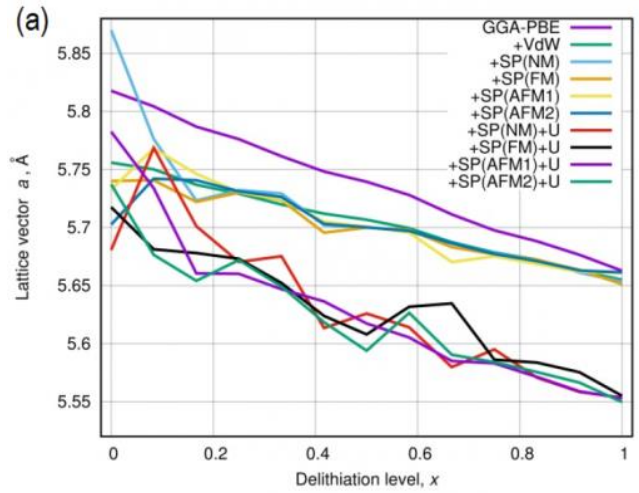

(b)

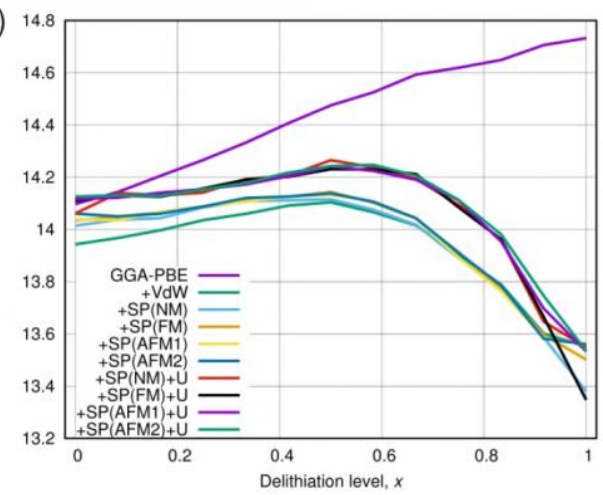

Fig. 1. The averaged dependencies of the LNO (a) $a$ and (b) $c$ lattice parameter values evaluated by means of the DFT calculations using formula (1) at the $\mathrm{T} \rightarrow \infty$ limit. 
For this reason, only the GGA-PBE together with its dispersion corrected (+vdW) models were used for the modeling of the $\mathrm{N}_{10} \mathrm{C}_{1} \mathrm{~A}_{1}$ configurational space. For the GGAPBE model, the relaxed structures, their energies and statistical weights are used for calculation by formula (1) of the average behavior of lattice parameters shown in Fig. 2a, where they are compared to the quantities observed from the in situ neutron diffraction experiments [12].
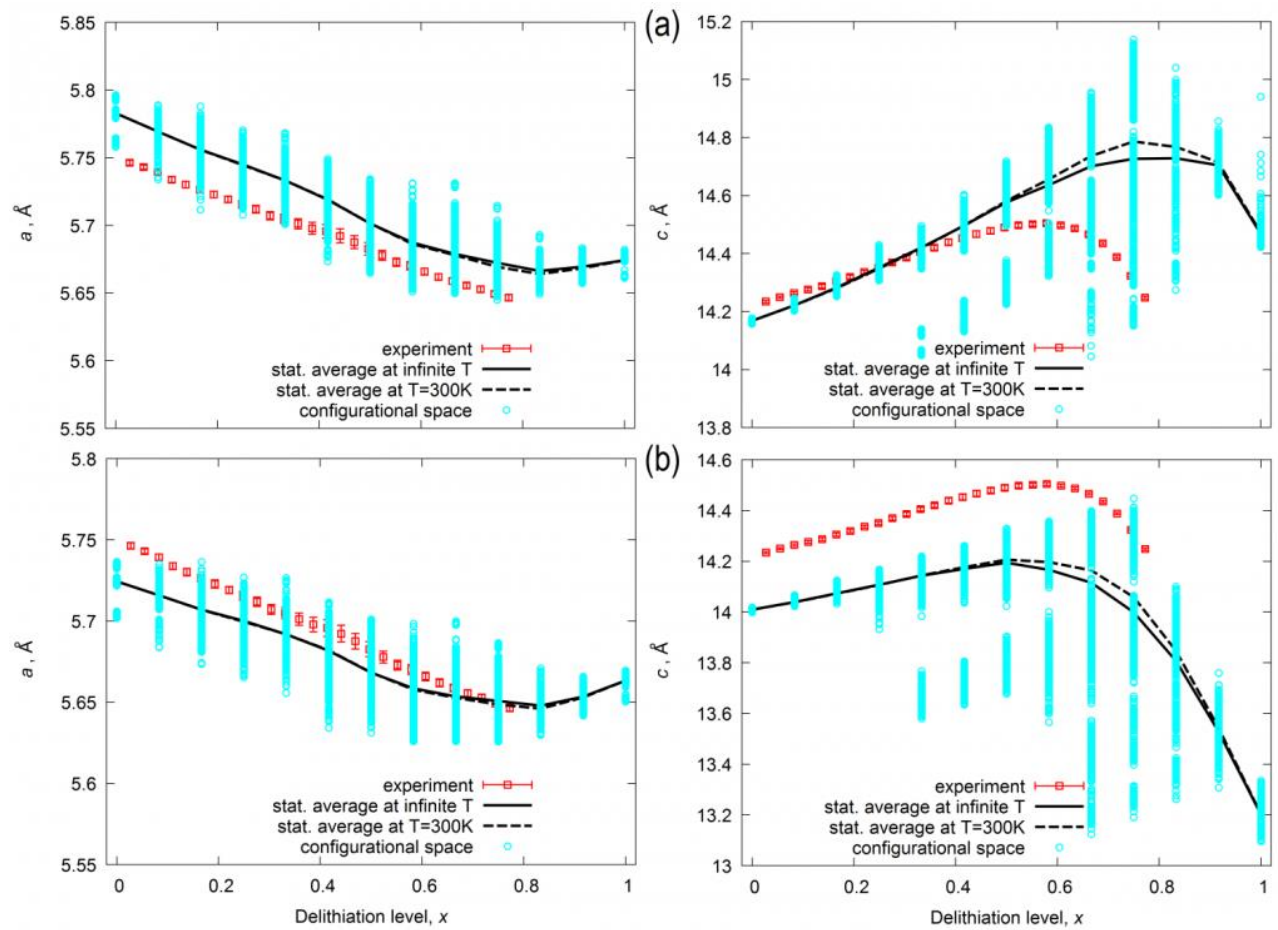

Fig. 2. Comparison of in situ neutron diffraction data for $a$ (left) and $c$ (right) lattice parameters of the NCA structure (squares) with the dependencies computed for the configurational space relaxed within the (a) GGA-PBE and (b) GGA-PBE-vdW models of $\mathrm{N}_{10} \mathrm{C}_{1} \mathrm{~A}_{1}$ (circles). An averaging is performed by formula (1) with (at temperature of $300 \mathrm{~K}$ ) and without accounts for energy balance of the configurational space.

The $a$ lattice parameter decreases at the initial stage of delithiation and increases after $x \approx 0.8$. This observation is in a good agreement with $\mathrm{x}$-ray diffraction data for deepcharged states of the NCA cathode [10]. Even a more pronounced effect is observed for the mainly increasing dependency of $c$ parameter, which starts to decrease at the latter stage of delithiation $(x=0.75 \div 0.80)$. According to the model applied, the described structural stabilization of the cathode can be only associated with doping.

The dependencies of the $a$ and $c$ lattice parameter values computed by means of the GGA-PBE-vdW model are depicted in Fig. $2 b$ and are much better consistent with the in situ neutron diffraction results. Based on the results of modeling of the LNO configurational space (Fig. 1), further improvement of the DFT model (by applying of the $+\mathrm{SP}$ and $+\mathrm{U}$ corrections) is expected to reduce the deviations for $c$ lattice parameter. On the other hand, the corresponding deviation of $a$ values should become higher. For these reasons, the subsequent extension of the DFT models was not considered. 
For the computed $c$ parameter dependencies the averaging at the finite temperature $(T=300 \mathrm{~K})$ and pure statistic averaging $(T \rightarrow \infty)$ deviate in the $x$ range of 0.6 to 0.8 (cf Fig. 2 $a$ and $b$ ). This fact is caused by the presence of the configurations with two fully empty Li layers in the structure [13]. The $a$ parameter behavior does not depend on the averaging method for the both models studied.

\section{Conclusions and remarks}

For the cathode material studied the doping effect results in non-monotonic behavior of the structural parameters $(a$ and $c$ ) at the latest stages of delithiation. In contrast, based on the results of LNO and $\mathrm{N}_{10} \mathrm{C}_{1} \mathrm{~A}_{1}$ modeling, the dispersion interactions significantly stabilize cathode material structure (only $c$ parameter) starting at $x \approx 0.5$. The proposed approach of the configurational space sampling seems to be promising tool for the computational investigation of the cathode materials. The development of the modeling approaches allows either refining of the experimental data or even predicting of the properties of perspective materials, avoiding costly and long-term synthesis, post-synthetic treatment and subsequent experimental studying. Possibility of a complement use of the full-configurational-spaces approaches with machine learning (regression) algorithms can significantly reduce a computational complexity of cathode materials investigations and, consequently, increase a predictive power of the modeling methods.

We are obliged to computational facilities of the Samara University complexes, namely, 'Sergey Korolev' and 'Zeolite' (Samara Center for Theoretical Materials Science). The computational studies were supported by the Russian Federation Government (projects No.14.B25.31.0005 and No.3.6588.2017/9.10). The work on the neutron diffraction experiments was supported by Russian Science Foundation (project No. 15-13-10006).

\section{References}

1. V. L. Chevrier, L. Liu et al. J. Electrochem. Soc. 161, A783-A791 (2014).

2. M. N. Obrovac, V. L. Chevrier Chem. Rev. 114, 11444-11502 (2014).

3. B. Xu, D. Qian et al. Mater. Sci. Eng. R Reports 73, 51-65 (2012).

4. E. V. Antipov, N. R. Khasanova, S. S. Fedotov IUCrJ 2, 85-94 (2015).

5. M. D. Bhatt, C. O’Dwyer Phys. Chem. Chem. Phys. 17, 4799-4844 (2015).

6. M. Aykol, S. Kim, C. Wolverton J. Phys. Chem. C 119, 19053-19058 (2015).

7. Z. Yang, Z. Zhang et al. J. Solid State Chem. 244, 52-60 (2016).

8. M. Dixit, M. Kosa et al. Phys. Chem. Chem. Phys. 18, 6799-6812 (2016).

9. F. Kong, C. Liang et al. Chem. Mater. 28, 6942-6952 (2016).

10. R. Robert, C. Bunzli et al. Chem. Mater. 27, 526-536 (2015).

11. W. K. Pang, M. Alam et al. J. Mater. Res. 30, 373-380 (2014).

12. I.A. Bobrikov, N.Yu. Samoylova et al. J. Power Sources 372, 74-81 (2017).

13. R.A. Eremin, P.N. Zolotarev et al. J. Phys. Chem. C 121, 28293-28305 (2017).

14. V.A. Blatov, A.P. Shevchenko, D.M. Proserpio Cryst. Growth Des. 14, 3576-3586 (2014).

15. G. Kresse, J. Furthmüller Phys. Rev. B 54, 11169-11186 (1996).

16. S. Grimme, J. Antony et al. J. Chem. Phys. 132, 154104 (2010).

17. A. Jain, G. Hautier et al.. Phys. Rev. B 84, 045115 (2011).

18. R. Grau-Crespo, S. Hamad et al. J. Phys. Condens. Matter 19, 256201 (2007). 